\title{
A normalization formula for the Jack polynomials in superspace and an identity on partitions
}

\author{
Luc Lapointe* \\ Instituto de Matemática y Física, Universidad de Talca \\ Casilla 747, Talca, Chile \\ lapointe@inst-mat.utalca.cl \\ Yvan Le Borgne ${ }^{\dagger}$ \\ CNRS, LaBRI, Université de Bordeaux 1 \\ 351 Cours de la Libération, 33405 Talence Cedex, France \\ yvan. leborgne@labri.fr \\ Philippe Nadeau ${ }^{\ddagger}$ \\ Fakultät für Mathematik, Universität Wien \\ Nordbergstraße 15, 1090 Vienna, Austria \\ philippe.nadeau@univie.ac.at \\ Submitted: Jan 28, 2008; Accepted: May 27, 2009; Published: Jun 5, 2009 \\ Mathematics Subject Classification: 05A15, 05E05

\begin{abstract}
We prove a conjecture of [3] giving a closed form formula for the norm of the Jack polynomials in superspace with respect to a certain scalar product. The proof is mainly combinatorial and relies on the explicit expression in terms of admissible tableaux of the non-symmetric Jack polynomials. In the final step of the proof appears an identity on weighted sums of partitions that we demonstrate using the methods of Gessel-Viennot.
\end{abstract}

${ }^{*}$ L. L. was partially supported by the Anillo Ecuaciones Asociadas a Reticulados financed by the World Bank through the Programa Bicentenario de Ciencia y Tecnología, and by the Programa Reticulados y Ecuaciones of the Universidad de Talca.

†Y.L.B. was partially supported by the French Agence Nationale de la Recherche, projects SADA ANR-05-BLAN-0372 and MARS ANR-06-BLAN-0193.

${ }_{\ddagger}^{\ddagger}$ P.N. was supported by the Austrian Science Foundation FWF, grant S9607-N13, in the framework of the National Research Network "Analytic Combinatorics and Probabilistic Number Theory". 


\section{Introduction}

Let $(x, \theta)=\left(x_{1}, \cdots x_{N}, \theta_{1}, \cdots \theta_{N}\right)$ be a collection of $2 N$ variables, called respectively bosonic and fermionic (or anticommuting or Grassmannian), obeying the relations

$$
x_{i} x_{j}=x_{j} x_{i}, \quad x_{i} \theta_{j}=\theta_{j} x_{i} \quad \text { and } \quad \theta_{i} \theta_{j}=-\theta_{j} \theta_{i} \quad\left(\Rightarrow \theta_{i}^{2}=0\right) .
$$

We call symmetric functions in superspace the ring of polynomials in these variables over the field $\mathbb{Q}$ that are invariant under the simultaneous interchange of $x_{i} \leftrightarrow x_{j}$ and $\theta_{i} \leftrightarrow \theta_{j}$ for any $i, j$. That is, defining

$$
\mathcal{K}_{\sigma} f\left(x_{1}, \ldots, x_{N}, \theta_{1}, \ldots, \theta_{N}\right):=f\left(x_{\sigma(1)}, \ldots, x_{\sigma(N)}, \theta_{\sigma(1)}, \ldots, \theta_{\sigma(N)}\right), \quad \sigma \in S_{N},
$$

we have that a polynomial $f\left(x_{1}, \ldots, x_{N}, \theta_{1}, \ldots, \theta_{N}\right)$ is a symmetric function in superspace iff

$$
\mathcal{K}_{\sigma} f\left(x_{1}, \ldots, x_{N}, \theta_{1}, \ldots, \theta_{N}\right)=f\left(x_{1}, \ldots, x_{N}, \theta_{1}, \ldots, \theta_{N}\right)
$$

for all permutations $\sigma$ in the symmetric group $S_{N}$.

Bases of the ring of symmetric functions in superspace can be indexed by superpartitions. A superpartition $\Lambda$ is of the form

$$
\Lambda:=\left(\Lambda^{a} ; \Lambda^{s}\right)=\left(\Lambda_{1}, \ldots, \Lambda_{m} ; \Lambda_{m+1}, \ldots, \Lambda_{N}\right)
$$

where

$$
\Lambda_{1}>\Lambda_{2}>\cdots>\Lambda_{m} \geqslant 0 \quad \text { and } \quad \Lambda_{m+1} \geqslant \Lambda_{m+2} \geqslant \cdots \geqslant \Lambda_{N} \geqslant 0 .
$$

In other words, $\Lambda^{a}$ is a partition with distinct parts (one of them possibly equal to zero), and $\Lambda^{s}$ is an ordinary partition. The degree of $\Lambda$ is $|\Lambda|=\Lambda_{1}+\cdots+\Lambda_{N}$ while its fermionic degree is $m$. The length $\ell(\Lambda)$ of $\Lambda$ is $m+\ell\left(\Lambda^{s}\right)$, where $\ell\left(\Lambda^{s}\right)$ is the number of non-zero parts in the partition $\Lambda^{s}$ (the usual length of a partition). Given a fixed degree $n$ and fermionic degree $m$, a superpartition that will be especially relevant for this work is

$$
\Lambda_{\min }:=\left(\delta_{m} ; 1^{\ell_{n, m}}\right),
$$

where

$$
\delta_{m}:=(m-1, m-2, \ldots, 0) \quad \text { and } \quad \ell_{n, m}:=n-\frac{m(m-1)}{2} .
$$

The superpartition $\Lambda_{\min }$ is the minimal one among the superpartitions of degree $n$ and fermionic degree $m$ in some order on superpartitions generalizing the dominance order on partitions (see [3]). Note that it will always be clear from the context what $n$ and $m$ are.

A natural basis for the ring of symmetric functions in superspace is given by the monomial functions:

$$
m_{\Lambda}=\frac{1}{f_{\Lambda^{s}}} \sum_{\sigma \in S_{N}} \mathcal{K}_{\sigma} \theta_{1} \cdots \theta_{m} x^{\Lambda},
$$


where

$$
x^{\Lambda}:=x_{1}^{\Lambda_{1}} \cdots x_{m}^{\Lambda_{m}} x_{m+1}^{\Lambda_{m+1}} \cdots x_{N}^{\Lambda_{N}}
$$

and

$$
f_{\Lambda^{s}}=\prod_{i \geqslant 0} m_{i}\left(\Lambda^{s}\right) !
$$

with $m_{i}\left(\Lambda^{s}\right)$ the number of $i$ 's in the partition $\Lambda^{s}$.

A less trivial basis of the the ring of symmetric functions in superspace is given by the Jack polynomials in superspace, $J_{\Lambda}$, which generalize the usual Jack polynomials. These polynomials, depending on a parameter $\alpha$, arose as eigenfunctions of a supersymmetric quantum-mechanical many-body problem. An explicit definition of the Jack polynomials in superspace involving non-symmetric Jack polynomials will be given in Section 2.3.

The main point of this article is to prove a conjecture, stated in [3], giving an explicit expression for the coefficient $c_{\Lambda}^{\min }(\alpha)$ of $\tilde{m}_{\Lambda_{\min }}:=\left(\ell_{n, m} !\right) m_{\Lambda_{\min }}$ in $J_{\Lambda}$, where $n=|\Lambda|$ and $m$ is the fermionic degree of $\Lambda$ (see Proposition 3). The relevance of this conjecture is that it gives as a corollary an explicit form for the norm of the Jack polynomials in superspace with respect to a certain scalar product. To be more precise, for a superpartition $\Lambda$, let the corresponding power sum products in superspace be given by

$$
p_{\Lambda}:=\tilde{p}_{\Lambda_{1}} \ldots \tilde{p}_{\Lambda_{m}} p_{\Lambda_{m+1}} \cdots p_{\Lambda_{N}} \quad \text { with } \quad p_{n}:=m_{(; n)} \quad \text { and } \quad \tilde{p}_{k}:=m_{(k ; 0)},
$$

and define the scalar product:

$$
\left\langle\left\langle p_{\Lambda} \mid p_{\Omega}\right\rangle_{\alpha}:=(-1)^{m(m-1) / 2} z_{\Lambda}(\alpha) \delta_{\Lambda, \Omega}, \quad z_{\Lambda}(\alpha):=\alpha^{\ell(\Lambda)} \prod_{i \geqslant 1} i^{m_{i}\left(\Lambda^{s}\right)} m_{i}\left(\Lambda^{s}\right) !\right.
$$

As shown in [3], the Jack polynomials in superspace are such that

$$
\left\langle\left\langle J_{\Lambda} \mid J_{\Omega}\right\rangle_{\alpha}=\alpha^{m+\ell_{n, m}} \frac{c_{\Lambda}^{\min }(\alpha)}{c_{\Lambda^{\prime}}^{\min }(1 / \alpha)} \delta_{\Lambda, \Omega},\right.
$$

where $\Lambda^{\prime}$, the conjugate of $\Lambda$, will be described at the end of Section 2.1. Obtaining an explicit expression for $c_{\Lambda}^{\min }(\alpha)$ thus immediately gives a closed form for the norm of the Jack polynomials in superspace with respect to this scalar product. We should point out that these results are natural analogs of classical results on Jack polynomials (see for instance [7]).

The proof of Proposition 3 relies on the explicit expressions for non-symmetric Jack polynomials in terms of admissible tableaux given in [4]. An interesting by-product of the proof is that it leads to an identity on partitions (see Identity 10) that we believe is worth stating here in the special case $\gamma=0^{m-1}$.

Identity 1 . For $i=1, \ldots, m$, let $\lambda^{(i)}$ be a partition of length $i$ with no parts larger than $m$. We say that $\lambda^{(1)}, \ldots, \lambda^{(m)}$ are non-intersecting if the $j$-th parts of $\lambda^{(j)}, \lambda^{(j+1)}, \ldots, \lambda^{(m)}$ are distinct for $j=1, \ldots, m$. In particular, this implies that $\left[\lambda_{1}^{(1)}, \ldots, \lambda_{1}^{(m)}\right]$ is a permutation 
in $S_{m}$. We define $\mathcal{V}_{0}$ to be the set of $\left(\lambda^{(1)}, \ldots, \lambda^{(m)}\right)$ such that $\lambda^{(1)}, \ldots, \lambda^{(m)}$ are nonintersecting. We say that $(i, j)$ is critical in $\left(\lambda^{(1)}, \ldots, \lambda^{(m)}\right) \in \mathcal{V}_{0}$ if $i \geqslant j \geqslant 2$ and $\lambda_{j}^{(i)}=\lambda_{j-1}^{(i)}$. Finally, let $a_{1}, \ldots, a_{m}$ and $b_{1}, \ldots, b_{m-1}$ be indeterminates. We have

$$
\prod_{1 \leqslant j<i \leqslant m}\left(a_{i}+1-a_{j}\right)=\sum_{\left(\lambda^{(1)}, \ldots, \lambda^{(m)}\right) \in \mathcal{V}_{0}} \operatorname{sgn}\left(\left[\lambda_{1}^{(1)}, \ldots, \lambda_{1}^{(m)}\right]\right) \prod_{(i, j) \text { critical }}\left(a_{\lambda_{j}^{(i)}}+b_{j-1}\right) .
$$

Observe that the L.H.S. does not depend on the $b_{i}$ 's while the R.H.S. does. The proof we provide of this identity relies crucially on the identification of the R.H.S. of (3) as a determinant using the methods of Gessel-Viennot [5].

\section{Definitions}

\subsection{Superpartitions}

Superpartitions were defined in the introduction. We describe here a diagrammatic representation of superpartitions that extends the notion of Ferrers' diagram. Recall [7] that the Ferrers' diagram of the partition $\lambda=\left(\lambda_{1}, \ldots, \lambda_{r}\right)$ is the set of cells in $\mathbb{Z}_{\geqslant 1}^{2}$ such that $1 \leqslant i \leqslant r$ and $1 \leqslant j \leqslant \lambda_{i}$. We use here the convention in which $i$ increases as one goes down. For instance, to $\lambda=(5,3,1,1)$ corresponds the diagram

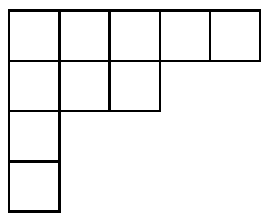

To every superpartition $\Lambda$, we can associate a unique partition $\Lambda^{*}$ obtained by deleting the semicolon and reordering the parts in non-increasing order. The diagram associated

to $\Lambda$, denoted by $D[\Lambda]$, is obtained by first drawing the Ferrers' diagram associated to $\Lambda^{*}$ and then adding a circle at the end of each row corresponding to an entry of $\Lambda^{a}$. If an entry of $\Lambda^{a}$ coincides with some entries of $\Lambda^{s}$, the row corresponding to that entry in $D[\Lambda]$ is considered to be the topmost one. For instance, if $\Lambda=(3,1,0 ; 5,3,2)$, we have $\Lambda^{*}=(5,3,3,2,1,0)$, and thus

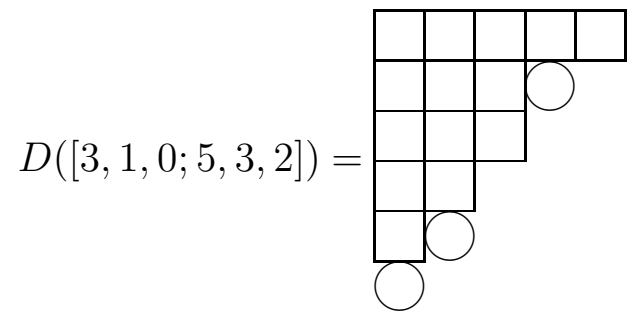

Note that with this definition, if the circles are considered as cells then $D[\Lambda]$ is still a partition. It is thus natural to define $\Lambda^{\prime}$, the conjugate of $\Lambda$, to be the superpartition obtained by transposing the diagram of $D[\Lambda]$ with respect to the main diagonal. Using the example above, one easily sees that $(3,1,0 ; 5,3,2)^{\prime}=(5,4,1 ; 3,1)$. 


\subsection{Non-symmetric Jack polynomials}

The non-symmetric Jack polynomials were first studied in [8] (although they had appeared before in physics as eigenfunctions of certain Dunkl-type operators [1]). These are polynomials $E_{\eta}(x ; \alpha)$ in a given number $N$ of variables $x=x_{1}, \ldots, x_{N}$, depending on a formal parameter $\alpha$ and indexed by compositions. For our purposes, we will reproduce the explicit combinatorial formula given in [4]. Let $\eta \in \mathbb{Z}_{\geqslant 0}^{N}$ be a composition with $N$ parts (some of them possibly equal to zero). The diagram of $\eta$ is the set of cells in $\mathbb{Z}_{\geqslant 1}^{2}$ such that $1 \leqslant i \leqslant N$ and $1 \leqslant j \leqslant \eta_{i}$. For instance, if $\eta=(0,1,3,0,0,6,2,5)$, the diagram of $\eta$ is

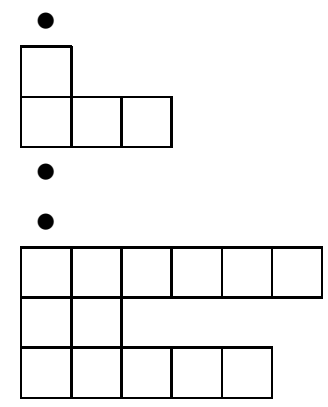

where a $\bullet$ represents an entry of length zero. For each cell $s=(i, j) \in \eta$, we define its arm-length $\mathbf{a}_{\eta}(s)$, leg-length $\mathbf{l}_{\eta}(s)$ and $\alpha$-hooklength $d_{\eta}(s)$ by:

$$
\begin{aligned}
\mathbf{a}_{\eta}(s) & =\eta_{i}-j \\
\mathbf{l}_{\eta}^{\prime}(s) & =\#\left\{k=1, \ldots, i-1 \mid j \leqslant \eta_{k}+1 \leqslant \eta_{i}\right\} \\
\mathbf{l}_{\eta}^{\prime \prime}(s) & =\#\left\{k=i+1, \ldots, N \mid j \leqslant \eta_{k} \leqslant \eta_{i}\right\} \\
\mathbf{l}_{\eta}(s) & =\mathbf{l}_{\eta}^{\prime}(s)+\mathbf{l}_{\eta}^{\prime \prime}(s) \\
d_{\eta}(s) & =\alpha\left(\mathbf{a}_{\eta}(s)+1\right)+\mathbf{l}_{\eta}(s)+1 .
\end{aligned}
$$

A diagrammatic representation of these parameters is provided in Figure 1. An explicit formula for $E_{\eta}(x ; \alpha)$ is given in terms of certain tableaux called 0 -admissible tableaux. A 0 -admissible tableau $T$ of shape $\eta$ is a filling of the cells of $\eta$ with letters belonging to $\{1,2, \ldots, N\}$ satisfying the following properties:

(1) There are never two identical letters in the same column;

(2) If the cell $(i, j)$ is filled with letter $c$, then a letter $c$ cannot occur in column $j+1$ in a row below row $i$;

(3) In the first column, a letter $i$ cannot occur in a row below row $i$.

A cell $(i, j)$ in a 0 -admissible tableau is called 0 -critical if either:

(a) $j>1$ and cell $(i, j-1)$ is filled with the same letter as cell $(i, j)$

(b) $j=1$ and cell $(i, j)=(i, 1)$ is filled with letter $i$. 


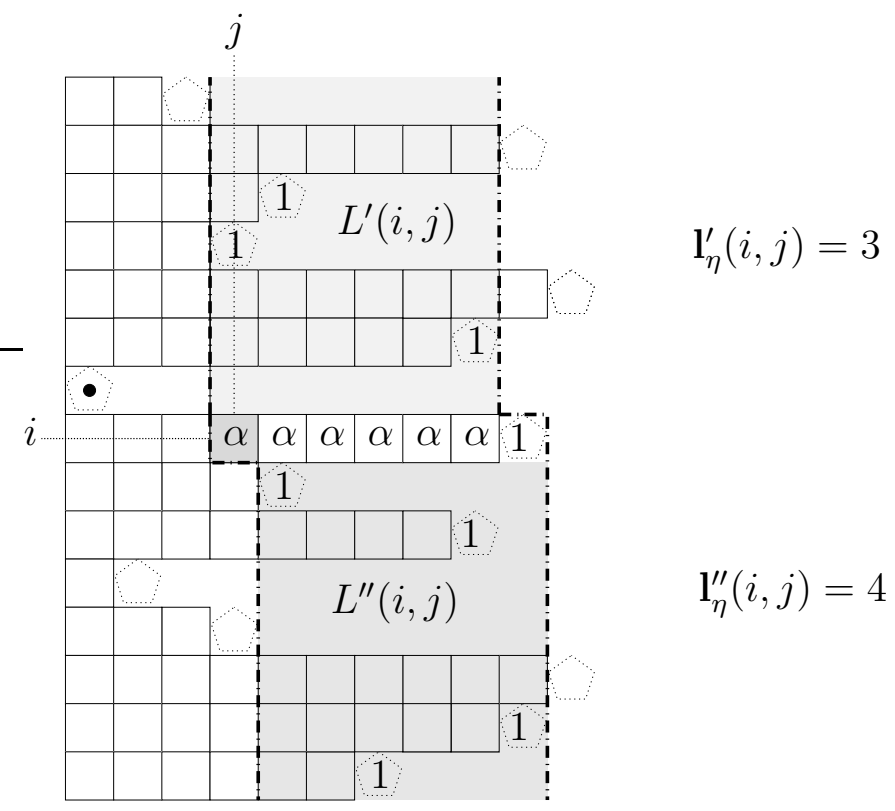

Figure 1: Diagrammatic representation of the $\alpha$-hooklength of the cell $s=(i, j)=(8,4)$. We add a (dotted) pentagonal cell at the end of each row. The three terms $1+\mathbf{l}_{\eta}^{\prime}(s)+$ $\mathbf{l}_{\eta}^{\prime \prime}(s)$ of the $\alpha$-hook length count respectively the pentagonal cell of row $i$, the number of pentagonal cells that belong to the set $L^{\prime}(s)=\left\{(k, l) \mid k<i\right.$ and $\left.j \leqslant l \leqslant \eta_{i}\right\}$ and the number of pentagonal cells that belong to $L^{\prime \prime}(s)=\left\{(k, l) \mid i<k\right.$ and $\left.j+1 \leqslant l \leqslant \eta_{i}+1\right\}$. The coefficient $\mathbf{a}_{\eta}(s)+1$ of $\alpha$ counts the cells in row $i$ from $(i, j)$ to $\left(i, \eta_{i}\right)$. In this example we have $d_{\eta}(s)=(1+3+4)+6 \alpha$.

Remark 2. As observed in [4], conditions (3) and (b) can be made superfluous if one defines a tableau $T^{0}$ obtained from $T$ by adding a column 0 filled with an $i$ in row $i$ for $i=1, \ldots, N$. In this case $T$ is 0 -admissible if $T^{0}$ satisfies (1) and (2). And $s$ is 0 -critical if it satisfies (a) when considered in $T^{0}$.

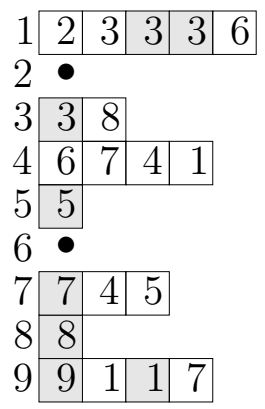

Figure 2: Example of a 0-admissible tableau. A column 0 has been added and the 0 -critical cells are shaded. 


\section{Defining}

$$
d_{T}^{0}(\alpha)=\prod_{s 0 \text {-critical }} d_{\eta}(s),
$$

the combinatorial formula for the non-symmetric Jack polynomials is given by

$$
E_{\eta}(x ; \alpha)=\left(\frac{1}{\prod_{s \in \eta} d_{\eta}(s)}\right) \sum_{T \text { 0-admissible of shape } \eta} d_{T}^{0}(\alpha) x^{\operatorname{ev}(T)},
$$

where ev $(T)$, the evaluation of $T$, is given by the vector $\left(|T|_{1}, \ldots,|T|_{N}\right)$ with $|T|_{i}$ the number of $i$ 's in the 0-admissible tableau $T$.

\subsection{Jack polynomials in superspace}

Given a superpartition $\Lambda=\left(\Lambda_{1}, \ldots, \Lambda_{m} ; \Lambda_{m+1}, \ldots, \Lambda_{N}\right)$ define $\tilde{\Lambda}$ to be the composition

$$
\tilde{\Lambda}:=\left(\Lambda_{m}, \ldots, \Lambda_{1}, \Lambda_{N}, \ldots, \Lambda_{m+1}\right) .
$$

It was established in [2] that the Jack polynomials in superspace can be obtained from the non-symmetric Jack polynomials through the following relation:

$$
J_{\Lambda}=\frac{(-1)^{m(m-1) / 2}}{f_{\Lambda^{s}}} \sum_{w \in S_{N}} \mathcal{K}_{w} \theta_{1} \cdots \theta_{m} E_{\tilde{\Lambda}}(x ; \alpha),
$$

where $f_{\Lambda^{s}}$ was defined in (1) and $\mathcal{K}_{w}$ was defined at the beginning of the introduction. In this article, this will serve as our definition of Jack polynomials in superspace.

Note that the composition $\tilde{\Lambda}$ is of a very special form. Its first $m$ rows (resp. last $N-m$ rows) are strictly increasing (resp. weakly increasing). Diagrammatically, it is made of two partitions (the first one of which without repeated parts) drawn in the French notation (largest row in the bottom). For instance if $\Lambda=(3,1,0 ; 5,3,3,0,0)$, we have $\tilde{\Lambda}=(0,1,3,0,0,3,3,5)$ whose diagram is given by

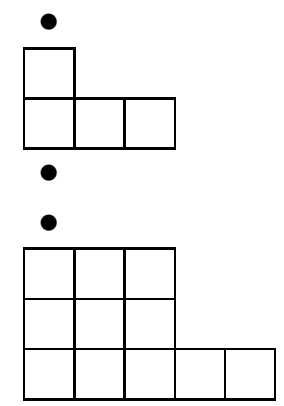

We will refer to the first $m$ rows (resp. last $N-m$ rows) of $\tilde{\Lambda}$ as the fermionic (resp. non-fermionic) portion of $\tilde{\Lambda}$. 


\section{The main result}

Given a cell $s$ in $D[\Lambda]$, let $a_{\Lambda}(s)$ be the number of cells (including the possible circle at the end of the row) to the right of $s$. Let also $\ell_{\Lambda}(s)$ be the number of cells (not including the possible circle at the bottom of the column) below $s$. Finally, let $\Lambda^{\circ}$ be the set of cells of $D[\Lambda]$ that do not appear at the same time in a row containing a circle and in a column containing a circle. The result we will prove in this article is the following, which was conjectured in [3].

Proposition 3. The coefficient $c_{\Lambda}^{\min }(\alpha)$ of $\tilde{m}_{\Lambda_{\min }}=\left(\ell_{n, m} !\right) m_{\Lambda_{\min }}$ in the monomial expansion of $J_{\Lambda}$ is given by

$$
c_{\Lambda}^{\min }(\alpha)=\frac{1}{\prod_{s \in \Lambda^{\circ}}\left(\alpha a_{\Lambda}(s)+\ell_{\Lambda}(s)+1\right)} .
$$

For instance, in the case $\Lambda=(3,1,0 ; 4,2,1)$, filling every cell $s \in \Lambda^{\circ}$ with the corresponding value $\left(\alpha a_{\Lambda}(s)+\ell_{\Lambda}(s)+1\right)$, we obtain

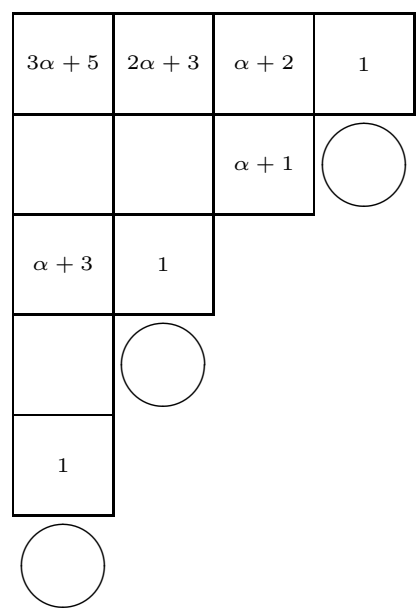

We thus get in this case

$$
c_{\Lambda}^{\min }(\alpha)=\frac{1}{(3 \alpha+5)(2 \alpha+3)(\alpha+2)(\alpha+1)(\alpha+3)} .
$$

\section{Derivation of the identity}

Combining (5) and (6), we have

$$
J_{\Lambda}=\frac{(-1)^{m(m-1) / 2}}{f_{\Lambda^{s}}}\left(\frac{1}{\prod_{s \in \tilde{\Lambda}} d_{\tilde{\Lambda}}(s)}\right) \sum_{w \in S_{N}} \mathcal{K}_{w} \theta_{1} \cdots \theta_{m} \sum_{T \text { 0-admissible }} d_{T}^{0}(\alpha) x^{\mathrm{ev}(T)},
$$

where the inner sum is over all 0 -admissible tableaux of shape $\tilde{\Lambda}$. 
To prove Proposition 3, we will compute the coefficient of $\tilde{m}_{\Lambda_{\min }}$ in the R.H.S. of (7) and show that it is as stated in the proposition. This will be done in a series of steps that will culminate at the end of the section with an identity on partitions. The identity will then be proven in the next section.

First, it is known [2] that a given expansion coefficient $c_{\Lambda \Omega}(\alpha)$ in

$$
J_{\Lambda}=\sum_{\Omega} c_{\Lambda \Omega}(\alpha) m_{\Omega}
$$

does not depend on the number of variables $N$ as long as $N \geqslant \ell(\Omega)$. Therefore, for simplicity we can set $N=\ell_{n, m}+m$ (which corresponds to $\ell\left(\Lambda_{\min }\right)$ ). Also, by symmetry, it is obvious that to compute the coefficient of $m_{\Lambda_{\text {min }}}$ it suffices to compute the coefficient of $\theta_{1} \cdots \theta_{m} x^{\Lambda_{\min }}$ in $J_{\Lambda}$.

In the remainder of this article, given a permutation $w, \operatorname{sgn}(w)$ will stand for the sign of the permutation $w$. Will will use $S_{m}$ and $S_{N-m}$ to stand for the subgroups of $S_{N}$ made out of elements permuting $\{1, \ldots, m\}$ and $\{m+1, \ldots, N\}$ respectively.

Lemma 4. T makes a non-zero contribution to the coefficient of $\theta_{1} \cdots \theta_{m} x^{\Lambda_{\min }}$ in the R.H.S. of $(7)$ iff $\operatorname{ev}(T)=\left(|T|_{1}, \ldots,|T|_{m}, 1, \ldots, 1\right)$ with $\left[|T|_{1}+1, \ldots,|T|_{m}+1\right]$ a permutation in $S_{m}$. Furthermore, if this is the case then we have $\mathcal{K}_{w} \theta_{1} \cdots \theta_{m} x^{\operatorname{ev}(T)}=$ $\pm \theta_{1} \cdots \theta_{m} x^{\Lambda_{\min }}$, where $w$ is of the form $w=w_{1} \times w_{2} \in S_{m} \times S_{N-m}$ with $w_{1}=[m-$ $\left.|T|_{1}, \ldots, m-|T|_{m}\right]$, in which case the sign \pm is given by $\operatorname{sgn}\left(w_{1}\right)$.

Proof. The first part of the lemma is obvious given that we must have $\left\{|T|_{1}, \ldots,|T|_{m}\right\}=$ $\{0,1, \ldots, m-1\}$ for $T$ to make a non-zero contribution to the coefficient of $\theta_{1} \cdots \theta_{m} x^{\Lambda_{\min }}$. The second part follows from the fact that the permutation $w$ must send $i$ to $m-|T|_{i}$, for all $i=1, \ldots, m$, in order to have $\mathcal{K}_{w} x^{\mathrm{ev}(T)}=x^{\Lambda_{\text {min }}}$. The sign arises from the anticommutation relations that the $\theta_{i}$ 's obey.

Given a tableau $T$, we denote by $T_{(m)}$ the subtableau made out of the cells of $T$ that are filled with letters from $\{1, \ldots, m\}$. We say that $P$ is a $\tilde{\Lambda}$-configuration if there exists a $T$ that makes a non-zero contribution to the coefficient of $\theta_{1} \cdots \theta_{m} x^{\Lambda_{\min }}$ in the R.H.S. of (7) such that $T_{(m)}=P$. Given a $\tilde{\Lambda}$-configuration $P$, we define $\mathcal{S}_{P}$ to be the set of 0 -admissible tableaux $T$ such that $T_{(m)}=P$. We let also

$$
d_{P}(\alpha):=\prod_{s 0 \text {-critical }} d_{\tilde{\Lambda}}(s)
$$

where a cell $s \in P$ is 0 -critical if it obeys the conditions (a) or (b) for a 0 -critical cell in a 0 -admissible tableau. Furthermore, let $\mathcal{C}_{\tilde{\Lambda}}$ be the set of $\tilde{\Lambda}$-configurations.

Lemma 5. Let $T \in \mathcal{S}_{P}$ for some $P \in \mathcal{C}_{\tilde{\Lambda}}$. Then

$$
d_{T}^{0}(\alpha)=d_{P}(\alpha) \prod_{i=N-\ell\left(\Lambda^{s}\right)+1}^{N} d_{\tilde{\Lambda}}((i, 1)) .
$$


Proof. There is exactly one occurrence of the letter $i$ in $T$ for $i=m+1, \ldots, N$ (recall that $\left.N=\ell_{n, m}+m\right)$. By condition (3) of the definition of 0-admissible tableaux, we must have a letter $N$ in position $(N, 1)$. Then cell $(N-1,1)$ must be filled with a letter $N-1$, since letter $N$ has already been used to fill cell $(N, 1)$. Applying this reasoning again and again we get that position $(i, 1)$, for $i=N-\ell\left(\Lambda^{s}\right)+1, \ldots, N$, is filled with a letter $i$. This implies that all these cells are 0 -critical and contribute to a factor $\prod_{i=N-\ell\left(\Lambda^{s}\right)+1}^{N} d_{\tilde{\Lambda}}((i, 1))$. From the definition of $d_{P}(\alpha)$, the contribution of the letters $1, \ldots, m$ in $d_{T}^{0}(\alpha)$ will be $d_{P}(\alpha)$. Finally, the remaining letters $m+1, \ldots, N-\ell\left(\Lambda^{s}\right)$ appear exactly once and cannot occupy positions $(i, 1)$ for $i=m+1, \ldots, N-\ell\left(\Lambda^{s}\right)$, since these cells do not belong to $\tilde{\Lambda}$. Therefore none of these letters occupies a 0 -critical position in $T$ and thus each of them contributes a factor 1 in $d_{T}^{0}(\alpha)$.

An easy consequence of the proof of the lemma is that the number of 0 -admissible tableaux in $\mathcal{S}_{P}$ is equal to $\left(\ell_{n, m}-\ell\left(\Lambda^{s}\right)\right)$ ! for any $\tilde{\Lambda}$-configuration $P$. Using Lemmas 4 and 5 , and defining $\operatorname{sgn}(P)$ to be the sign of the permutation $\left[m-|P|_{1}, \ldots, m-|P|_{m}\right]$, we then get from $(7)$ that

$\left.J_{\Lambda}\right|_{m_{\Lambda_{\min }}}=\frac{(-1)^{\frac{m(m-1)}{2}}}{f_{\Lambda^{s}}}\left(\frac{\prod_{i=N-\ell\left(\Lambda^{s}\right)+1}^{N} d_{\tilde{\Lambda}}((i, 1))}{\prod_{s \in \tilde{\Lambda}} d_{\tilde{\Lambda}}(s)}\right)\left(\ell_{n, m}-\ell\left(\Lambda^{s}\right)\right) ! \ell_{n, m} ! \sum_{P \in \mathcal{C}_{\tilde{\Lambda}}} \operatorname{sgn}(P) d_{P}(\alpha)$,

where $\ell_{n, m}$ ! accounts for the number of elements in $S_{N-m}$. As a consequence, in the monomial expansion of $J_{\Lambda}$, the coefficient $c_{\Lambda}^{\min }(\alpha)$ of $\tilde{m}_{\Lambda_{\min }}=\left(\ell_{n, m} !\right) m_{\Lambda_{\min }}$ is

$$
c_{\Lambda}^{\min }(\alpha)=\frac{(-1)^{m(m-1) / 2}}{f_{\Lambda^{s}}}\left(\frac{\prod_{i=N-\ell\left(\Lambda^{s}\right)+1}^{N} d_{\tilde{\Lambda}}((i, 1))}{\prod_{s \in \tilde{\Lambda}} d_{\tilde{\Lambda}}(s)}\right)\left(\ell_{n, m}-\ell\left(\Lambda^{s}\right)\right) ! \sum_{P \in \mathcal{C}_{\tilde{\Lambda}}} \operatorname{sgn}(P) d_{P}(\alpha) .
$$

The next lemma will further simplify this equation.

Lemma 6. We have

$$
\frac{\left(\prod_{s \in \tilde{\Lambda}} d_{\tilde{\Lambda}}(s)\right)\left(\prod_{i \geqslant 1} m_{i}\left(\Lambda^{s}\right) !\right)}{\prod_{s \in \Lambda^{\circ}}\left(\alpha a_{\Lambda}(s)+\ell_{\Lambda}(s)+1\right)}=\left(\prod_{i=N-\ell\left(\Lambda^{s}\right)+1}^{N} d_{\tilde{\Lambda}}((i, 1))\right)\left(\prod_{1 \leqslant j<i \leqslant m} d_{\tilde{\Lambda}}\left(\left(i, \tilde{\Lambda}_{j}+1\right)\right)\right) .
$$

Proof. The proof will proceed by cancellation of certain terms in the L.H.S. of the equation to obtain the R.H.S. Figure 3 illustrates the general idea of the proof.

Suppose $s=(i, j) \in \Lambda^{\circ}$ belongs to a fermionic row of $D[\Lambda]$ (one that ends with a circle). Then row $i$ of $D[\Lambda]$ corresponds to a row $k \in\{1, \ldots, m\}$ of $\tilde{\Lambda}$. We have then

$$
\alpha a_{\Lambda}((i, j))+\ell_{\Lambda}((i, j))+1=\alpha\left(\mathbf{a}_{\tilde{\Lambda}}((k, j))+1\right)+\mathbf{l}_{\tilde{\Lambda}}((k, j))+1=d_{\tilde{\Lambda}}((k, j)) .
$$

In this case $a_{\Lambda}((i, j))=\mathbf{a}_{\tilde{\Lambda}}((k, j))+1$ since both rows are of the same length and row $i$ of $D[\Lambda]$ has a circle (which accounts for the plus one). We also have that $\ell_{\Lambda}((i, j))=$ 

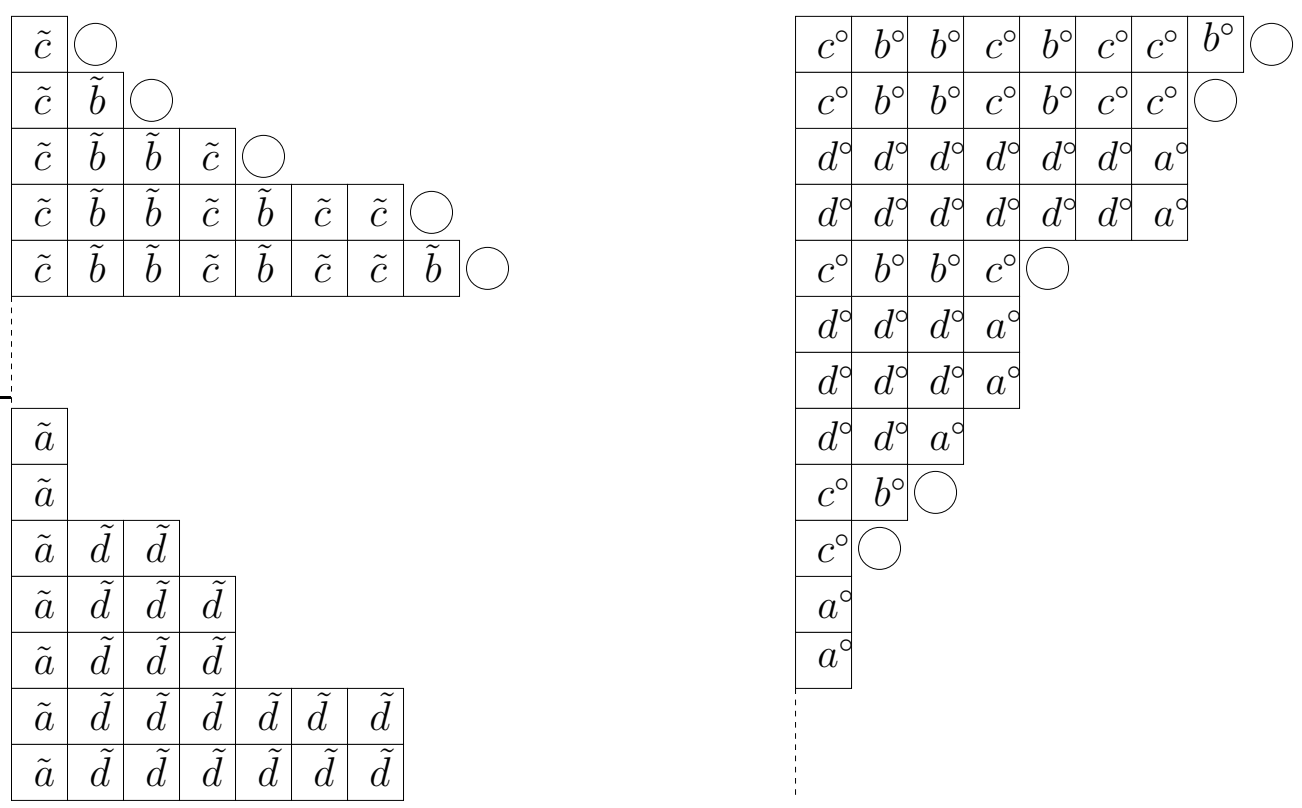

Figure 3: There is a weight preserving bijection between cells of $\{\tilde{c}, \tilde{d}\} \subset \tilde{\Lambda}$ and those of $\left\{c^{\circ}, d^{\circ}\right\} \subset \Lambda^{\circ} \subset \Lambda^{*}$. Roughly speaking, this bijection corresponds to a sorting of rows according to their length and a cyclic shift of one cell to the left for non-fermionic rows. We denote by $W(X)$ the product of the appropriate weight of the cells in $X$. The bijection implies then $W(\{\tilde{c}, \tilde{d}\})=W\left(\left\{c^{\circ}, d^{\circ}\right\}\right)$, and as a consequence we get $\frac{W(\tilde{\Lambda})}{W(\{\tilde{a}\}) W(\{\tilde{b}\})}=W(\{\tilde{c}, \tilde{d}\})=W\left(\left\{c^{\circ}, d^{\circ}\right\}\right)=\frac{W\left(\Lambda^{\circ}\right)}{W\left(\left\{a^{\circ}\right\}\right)}$.

$\mathbf{l}_{\tilde{\Lambda}}((k, j))$. This is because $\mathbf{l}_{\tilde{\Lambda}}^{\prime \prime}((k, j))$ (resp. $\left.\mathbf{l}_{\tilde{\Lambda}}^{\prime}((k, j))\right)$ accounts for the non-fermionic (resp. fermionic) rows that contribute to $\ell_{\Lambda}((i, j))$. The only way $\mathbf{l}_{\tilde{\Lambda}}^{\prime}$ would not correspond to the number of fermionic rows contributing to $\ell_{\Lambda}((i, j))$ is if some row above row $k$ in the diagram of $\tilde{\Lambda}$ was of length $j-1$ (in which case it would count one too many row). But this is not possible since this would imply that there is a circle in column $j$ of $D[\Lambda]$ and thus that $s \notin \Lambda^{\circ}$. Therefore (10) follows. Note that the cells that are not canceled in the first $m$ rows of $\tilde{\Lambda}$ are exactly the cells $\left(i, \tilde{\Lambda}_{j}+1\right)$, for $1 \leqslant j<i \leqslant m$, appearing in the R.H.S. of (9).

Suppose $(i, j) \in \Lambda^{\circ}$ does not belong to a fermionic row of $D[\Lambda]$ and does not lie at the end of its row. Then row $i$ of $D[\Lambda]$ corresponds to a row $k \in\left\{N-\ell\left(\Lambda^{s}\right)+1, \ldots, N\right\}$ of $\tilde{\Lambda}$. In this correspondence, if there are $p$ rows of the same length as row $i$ that do not end with a circle in $D[\Lambda]$ and row $i$ is the $r$-th one of them starting from the top, then we choose $k$ to be also the $r$-th one (also starting from the top) of that length in the fermionic portion of $\tilde{\Lambda}$. We have then

$$
\alpha a_{\Lambda}((i, j))+\ell_{\Lambda}((i, j))+1=\alpha\left(\mathbf{a}_{\tilde{\Lambda}}((k, j+1))+1\right)+\mathbf{l}_{\tilde{\Lambda}}((k, j+1))+1=d_{\tilde{\Lambda}}((k, j+1)) .
$$

It is easy to see that $a_{\Lambda}((i, j))=\mathbf{a}_{\tilde{\Lambda}}((k, j+1))+1$ since both rows are of the same length and row $i$ of $D[\Lambda]$ is not fermionic. We now need to see that $\ell_{\Lambda}((i, j))=\mathbf{l}_{\tilde{\Lambda}}((k, j+1))$. 
First, $\mathbf{l}_{\tilde{\Lambda}}^{\prime \prime}((k, j+1))$ accounts for all the rows below row $i$ of $D[\Lambda]$ of the same length as row $i$ and which contribute to $\ell_{\Lambda}((i, j))$. Then $\mathbf{l}_{\tilde{\Lambda}}^{\prime}((k, j+1))$ accounts for all the rows below row $i$ of $D[\Lambda]$ smaller than row $i$ that contribute to $\ell_{\Lambda}((i, j))$.

The cells in the fermionic portion of $\tilde{\Lambda}$ that are not canceled are those that lie in the first column and which correspond to the cells $(i, 1)$, for $i=N-\ell\left(\Lambda^{s}\right)+1, \ldots, N$, appearing in the R.H.S. of (9). And finally, the cells of $\Lambda^{\circ}$ that are not canceled are those lying at the end of a non-fermionic row. It is easy to see that their contribution is $\prod_{i \geqslant 1} m_{i}\left(\Lambda^{s}\right) !$

Using the previous lemma, equation (8), and the fact that

$$
f_{\Lambda^{s}}=\left(\ell_{n, m}-\ell\left(\Lambda^{s}\right)\right) ! \prod_{i \geqslant 1} m_{i}\left(\Lambda^{s}\right) !,
$$

we have

$$
c_{\Lambda}^{\min }(\alpha) \prod_{s \in \Lambda^{\circ}}\left(\alpha a_{\Lambda}(s)+\ell_{\Lambda}(s)+1\right)=\frac{(-1)^{m(m-1) / 2}}{\prod_{1 \leqslant j<i \leqslant m} d_{\tilde{\Lambda}}\left(\left(i, \tilde{\Lambda}_{j}+1\right)\right)} \sum_{P \in \mathcal{C}_{\tilde{\Lambda}}} \operatorname{sgn}(P) d_{P}(\alpha) .
$$

We will now see that it is not necessary to sum over all $P \in \mathcal{C}_{\tilde{\Lambda}}$. Let $\mathcal{G}_{\tilde{\Lambda}}$ be the set of all $\tilde{\Lambda}$-configurations $P$ such that for every $i=1, \ldots, m$ there is a letter $i$ in column $j$ of $P$ for $j=1, \ldots,|P|_{i}$. We will refer to $\mathcal{G}_{\tilde{\Lambda}}$ as the set of good $\tilde{\Lambda}$-configurations.

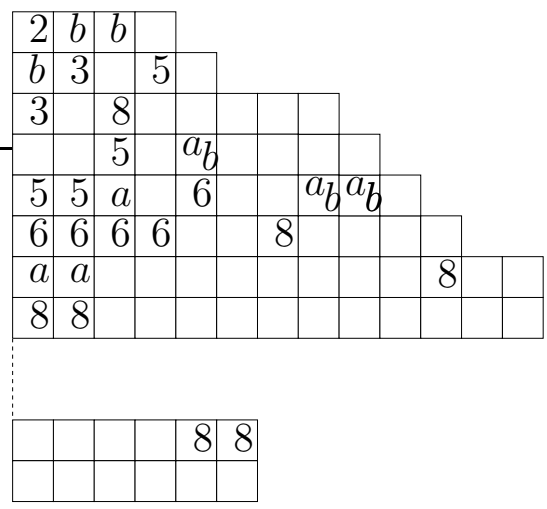

Figure 4: Here are two bad $\tilde{\Lambda}$-configurations mapped onto each others by the involution. Empty cells implicitly contain a label greater than $m$ (set equal to 8 in the example). In cells with two labels, the labels in the upper left (resp. lower right) corner correspond to the labels of $P\left(\right.$ resp. $\left.P^{\prime}\right)$. In the example, we have $a=7, j=4, b=4$. Observe that we can have labels not larger than $m$ in the non-fermionic portion of a $\tilde{\Lambda}$-configuration. For instance the 8 in column 5 is possible only because there is no 8 in column 4 .

Lemma 7. We have

$$
\sum_{P \in \mathcal{C}_{\tilde{\Lambda}}} \operatorname{sgn}(P) d_{P}(\alpha)=\sum_{P \in \mathcal{G}_{\tilde{\Lambda}}} \operatorname{sgn}(P) d_{P}(\alpha)
$$


Proof. The idea is to construct a sign-reversing involution among the $\tilde{\Lambda}$-configurations that do not belong to $\mathcal{G}_{\tilde{\Lambda}}$, which we will call bad $\tilde{\Lambda}$-configurations. Figure 4 illustrates the involution that follows. Let $P$ be a bad $\tilde{\Lambda}$-configuration. Let $j$ be the smallest integer such that there exists a letter $a$ that occurs in some column $j^{\prime}>j$ of $P$ but does not occur in column $j$ of $P$. If there are many such $a$ 's, pick the one such that $|P|_{a}$ is the smallest. Let $b$ be such that $|P|_{b}=j-1$. By definition the $b$ 's in $P$ occur exactly in the first $j-1$ columns. Therefore $P^{\prime}$ obtained from $P$ by replacing the $a$ 's that occur to the right of column $j$ with $b$ 's is also a bad $\tilde{\Lambda}$-configuration. We obviously have that $\operatorname{sign}\left(P^{\prime}\right)=-\operatorname{sgn}(P)$ and $d_{P^{\prime}}(\alpha)=d_{P}(\alpha)$. This operation is obviously an involution.

Now, suppose that $P$ is a good $\tilde{\Lambda}$-configuration, and fix an $i \in\{1, \ldots, m\}$. By the definition of a 0 -admissible tableau (recall that $P=T_{(m)}$ for some 0 -admissible tableau $T$ ), the letter $i$ in the first column of $P$ (if it exists) is in a row $i_{1} \leqslant i \leqslant m$. Again by the the definition of a 0 -admissible tableau, the letter $i$ in the second column of $P$ (if it exists) is in a row $i_{2} \leqslant i_{i} \leqslant i \leqslant m$. Using this argument again and again, we get that the letters $i$ in column $j=1, \ldots,|P|_{i}$ lie in a row $i_{j}$ such that $m \geqslant i \geqslant i_{1} \geqslant i_{2} \geqslant \cdots \geqslant i_{|P|}$. This gives the following lemma.

Lemma 8. $P$ is a good $\tilde{\Lambda}$-configuration iff $\left[|P|_{1}+1, \ldots,|P|_{m}+1\right]$ is a permutation of $S_{m}$ and the letters $i$ in column $j=1, \ldots,|P|_{i}$ lie in a row $i_{j}$ such that $m \geqslant i \geqslant i_{1} \geqslant i_{2} \geqslant$ $\cdots \geqslant i_{|P|_{i}}$. In particular, the cells in a good $\tilde{\Lambda}$-configuration all lie in the first $m$ rows of $\tilde{\Lambda}$, and thus the concept of good $\tilde{\Lambda}$-configuration only depends on the fermionic portion of $\tilde{\Lambda}$.

We will now see that there is an easy description of the $\alpha$-hooklengths of the cells in the fermionic portion of $\tilde{\Lambda}$. Let $v_{k}\left(\Lambda^{s}\right)$ be equal to the number of rows of $\Lambda^{s}$ that are smaller or equal to $k$. Then it is easy to see that we have, for $(i, j) \in \tilde{\Lambda}$ such that $1 \leqslant i \leqslant m$ :

$$
d_{\tilde{\Lambda}}((i, j))=\alpha\left(\tilde{\Lambda}_{i}-j+1\right)+\mathbf{l}_{\tilde{\Lambda}}^{\prime}((m, j))-(m-i)+v_{\tilde{\Lambda}_{i}}\left(\Lambda^{s}\right)-v_{j-1}\left(\Lambda^{s}\right)+1 .
$$

It proves convenient to write this equation as

$$
d_{\tilde{\Lambda}}((i, j))=a_{i}+b_{j},
$$

where $a_{i}=\alpha \tilde{\Lambda}_{i}+v_{\tilde{\Lambda}_{i}}\left(\Lambda^{s}\right)+i$ and $b_{j}=\alpha(1-j)+\mathbf{l}_{\tilde{\Lambda}}^{\prime}((m, j))-m-v_{j-1}\left(\Lambda^{s}\right)+1$. Note that we have

$$
b_{\tilde{\Lambda}_{j}+1}=1-a_{j},
$$

since $\mathbf{l}_{\tilde{\Lambda}}^{\prime}\left(\left(m, \tilde{\Lambda}_{j}+1\right)\right)=m-j$. This implies that

$$
(-1)^{m(m-1) / 2} \prod_{1 \leqslant j<i \leqslant m} d_{\tilde{\Lambda}}\left(\left(i, \tilde{\Lambda}_{j}+1\right)\right)=\prod_{1 \leqslant j<i \leqslant m}\left(a_{j}-a_{i}-1\right) .
$$

Using Lemma 7 and the previous equation, (12) becomes

$$
c_{\Lambda}^{\min }(\alpha) \prod_{s \in \Lambda^{\circ}}\left(\alpha a_{\Lambda}(s)+\ell_{\Lambda}(s)+1\right)=\frac{1}{\prod_{1 \leqslant j<i \leqslant m}\left(a_{j}-a_{i}-1\right)} \sum_{P \in \mathcal{G}_{\tilde{\Lambda}}} \operatorname{sgn}(P) d_{P},
$$


where

$$
d_{P}:=\prod_{(i, j) \in P ;(i, j)}\left(a_{i}+b_{j}\right) .
$$

First observe that only $b_{1}, \ldots, b_{m-1}$ will appear in $d_{P}$ since the definition of a good $\tilde{\Lambda}$ configuration $P$ implies that the cells of $P$ all lie within the first $m-1$ columns, as do all its 0 -critical cells. It is also natural to consider the $a_{i}$ 's and $b_{i}$ 's as general indeterminates rather than as the special expressions given after Equation (13). Therefore, Proposition 3 holds if the following identity holds.

Identity 9 (First form of the identity). Let $a_{1}, \ldots, a_{m}$ and $b_{1}, \ldots, b_{m-1}$ be indeterminates such that if $\tilde{\Lambda}_{i}<m-1$ then $b_{\tilde{\Lambda}_{i}+1}=1-a_{i}$. We have then

$$
\prod_{1 \leqslant j<i \leqslant m}\left(a_{j}-a_{i}-1\right)=\sum_{P \in \mathcal{G}_{\tilde{\Lambda}}} \operatorname{sgn}(P) d_{P},
$$

where we recall that the sum is over the set of good $\Lambda_{\min }$-configurations described in Lemma $8, \operatorname{sgn}(P)$ is the sign of the permutation $\left[m-|P|_{1}, \ldots, m-|P|_{m}\right]$, and $d_{P}$ was defined in (14).

This identity can be translated into the language of partitions. For $i=1, \ldots, m$, let $\lambda^{(i)}$ be a partition of length $i$ with no parts larger than $m$. We say that $\lambda^{(1)}, \ldots, \lambda^{(m)}$ are nonintersecting if the $j$-th parts of $\lambda^{(j)}, \lambda^{(j+1)}, \ldots, \lambda^{(m)}$ are distinct for $j=1, \ldots, m$. In particular, this implies that $\left[\lambda_{1}^{(1)}, \ldots, \lambda_{1}^{(m)}\right]$ is a permutation in $S_{m}$. Given $\gamma=\left(\gamma_{1}, \ldots, \gamma_{m-1}\right) \in$ $\{0,1\}^{m-1}$, we define $\mathcal{V}_{\gamma}$ to be the set of $\left(\lambda^{(1)}, \ldots, \lambda^{(m)}\right)$ such that $\lambda^{(1)}, \ldots, \lambda^{(m)}$ are nonintersecting and such that $\lambda_{j+1}^{(i)}>\#\left\{k \leqslant j \mid \gamma_{k}=1\right\}$ for all $i=j+1, \ldots, m$. Finally, we say that $(i, j)$ is critical in $\left(\lambda^{(1)}, \ldots, \lambda^{(m)}\right) \in \mathcal{V}_{\gamma}$ if $i \geqslant j \geqslant 2$ and $\lambda_{j}^{(i)}=\lambda_{j-1}^{(i)}$.

Identity 10 (Second form of the identity). Let $\gamma=\left(\gamma_{1}, \ldots, \gamma_{m-1}\right) \in\{0,1\}^{m-1}$. Let also $a_{1}, \ldots, a_{m}$ and $b_{1}, \ldots, b_{m-1}$ be indeterminates such that if $\gamma_{j}=1$ then $b_{j}=1-a_{r}$, where $r=\#\left\{k \leqslant j \mid \gamma_{k}=1\right\}$. We have then

$$
\prod_{1 \leqslant j<i \leqslant m}\left(a_{i}+1-a_{j}\right)=\sum_{\left(\lambda^{(1)}, \ldots, \lambda^{(m)}\right) \in \mathcal{V}_{\gamma}} \operatorname{sgn}\left(\left[\lambda_{1}^{(1)}, \ldots, \lambda_{1}^{(m)}\right]\right) \prod_{(i, j) \text { critical }}\left(a_{\lambda_{j}^{(i)}}+b_{j-1}\right),
$$

where the set $\mathcal{V}_{\gamma}$ was defined above.

Proof that Identity 9 and Identity 10 are equivalent. Let $\gamma_{j}=1$ iff there is a part of size $j-1$ in the fermionic portion of $\tilde{\Lambda}$. We thus have that $\tilde{\Lambda}_{i}<m-1$ iff $\gamma_{j}=1$ for $j=\tilde{\Lambda}_{i}+1$. In this case, $b_{\tilde{\Lambda}_{i}+1}=1-a_{i}$ is equivalent to $b_{j}=1-a_{r}$, with $r=\#\left\{k \leqslant j \mid \gamma_{k}=1\right\}$, given that $i$ is equal to the number of parts smaller or equal to $\Lambda_{i}$ in the fermionic portion of $\tilde{\Lambda}$. Note that $\gamma$ is only in bijection with the fermionic portion of $\tilde{\Lambda}$ whose parts are smaller than $m-1$. But since this is the only relevant part in Identity 9 , the relations between the $a_{i}$ 's and $b_{j}$ 's are the same. 

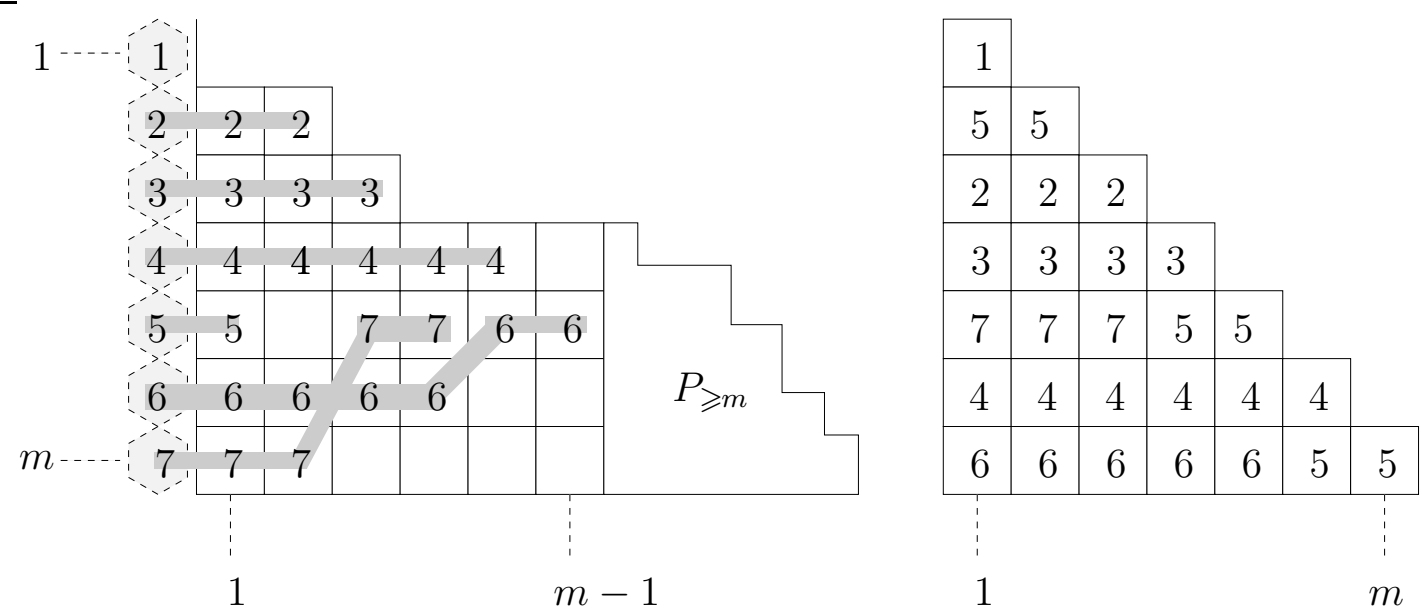

Figure 5: An example of the bijection between $\mathcal{G}_{\tilde{\Lambda}}$ and $\mathcal{V}_{\gamma}$ in the case $m=7$ and $\gamma=$ $(1,0,1,1,0,0)$. On the left, we draw the diagrammatic representation of the relevant part of the good- $\tilde{\Lambda}$ configuration $P$ and an additional column 0 of hexagons labeled by the rows' indices. Cells to the right of column $m-1$ define a subconfiguration $P_{\geqslant m}$ whose shape or labels do not contribute to the weight. On the right, we have the element of $\mathcal{V}_{\gamma}$ on which is mapped this configuration. In the configuration, the thick grey line starting from the hexagon labeled by $i$ represents the row $\lambda^{\left(k_{i}+1\right)}=\left(i, i_{1}, i_{2}, \ldots i_{k_{i}}\right)$ in the partition.

We now show that there is a bijection between the sets $\mathcal{G}_{\tilde{\Lambda}}$ and $\mathcal{V}_{\gamma}$. Let $P \in \mathcal{G}_{\tilde{\Lambda}}$. Suppose that letter $i$ is such that $\left|P_{i}\right|=k_{i}$ (that is, letter $i$ occurs $k_{i}$ times in $P$ ). From Lemma 8, this implies that letter $i$ appears in columns $1, \ldots, k_{i}$ in positions $i_{1}, \ldots, i_{k_{i}}$ such that $m \geqslant i \geqslant i_{1} \geqslant i_{2} \geqslant \cdots \geqslant i_{k_{i}}$. This gives us a partition $\lambda^{\left(k_{i}+1\right)}=\left(i, i_{i}, i_{2}, \ldots, i_{k_{i}}\right)$ of length $k_{i}+1$ with no parts larger than $m$. If we do the same for $i=1, \ldots, m$ we obtain partitions $\lambda^{(1)}, \ldots, \lambda^{(m)}$ that are non-intersecting since the $i_{j}$ 's are distinct for a fixed $j$ (given that no two letters can occupy the same cell). Furthermore, if $j>\tilde{\Lambda}_{i}$ then cell $(i, j)$ is not in $P$. The only rows $l$ that are allowed in column $j$ are thus those such that $l>\#\left\{k \leqslant j \mid \gamma_{k}=1\right\}$. Since the cell $(l, j)$ in $P$ corresponds to the $(j+1)$-th part of $\lambda^{(i)}$ for some $i$, we have the condition $\lambda_{j+1}^{(i)}>\#\left\{k \leqslant j \mid \gamma_{k}=1\right\}$ for all $i=j+1, \ldots, m$. Given a $\left(\lambda^{(1)}, \ldots, \lambda^{(m)}\right) \in \mathcal{V}_{\gamma}$, one can easily reconstruct the corresponding $P \in \mathcal{G}_{\tilde{\Lambda}}$ by reversing the procedure we just described. Figure 5 provides an example of the bijection we just constructed.

If $P \longleftrightarrow\left(\lambda^{(1)}, \ldots, \lambda^{(m)}\right)$ in the bijection, the permutation $\left[\lambda_{1}^{(1)}, \ldots, \lambda_{1}^{(m)}\right]$ is the inverse of the permutation $\left[|P|_{1}+1, \ldots,|P|_{m}+1\right]$ since in the bijection $\lambda_{1}^{(j)}=i$ iff $|P|_{i}+1=j$. This implies that

$$
\operatorname{sgn}\left(\left[\lambda_{1}^{(1)}, \ldots, \lambda_{1}^{(m)}\right]\right)=\operatorname{sgn}\left(\left[|P|_{1}+1, \ldots,|P|_{m}+1\right]\right),
$$

given that $\operatorname{sgn}(w)=\operatorname{sgn}\left(w^{-1}\right)$ for any permutation $w$. Since

$$
\operatorname{sgn}\left(\left[|P|_{1}+1, \ldots,|P|_{m}+1\right]\right)=(-1)^{m(m-1) / 2} \operatorname{sgn}\left(\left[m-|P|_{1}, \ldots, m-|P|_{m}\right]\right),
$$


this takes into account the changes from $\left(a_{j}-a_{i}-1\right)$ to $\left(a_{i}+1-a_{j}\right)$ in the L.H.S. of the identity.

Finally, we have that

$$
\prod_{\left(i^{\prime}, j^{\prime}\right) \in P ;\left(i^{\prime}, j^{\prime}\right)}\left(a_{i^{\prime}}+b_{j^{\prime}}\right)=\prod_{(i, j) \text { critical }}\left(a_{\lambda_{j}^{(i)}}+b_{j-1}\right) .
$$

This is seen in the following way. Observing that cell $\left(i^{\prime}, j^{\prime}\right)$ of $P$, when filled with an integer, corresponds in the bijection to a $\lambda_{j=j^{\prime}+1}^{(i)}$ for some $i \geqslant j$, we have that $\left(a_{i^{\prime}}+b_{j^{\prime}}\right)=$ $\left(a_{\lambda_{j}^{(i)}}+b_{j-1}\right)$. Then recall that $\left(i^{\prime}, j^{\prime}\right)$ is 0 -critical iff $\left(\right.$ a) $j^{\prime}>1$ and $\left(i^{\prime}, j^{\prime}-1\right)$ is filled with the same letter as $\left(i^{\prime}, j^{\prime}\right)$ or (b) $j^{\prime}=1$ and $\left(i^{\prime}, j^{\prime}\right)=\left(i^{\prime}, 1\right)$ is filled with an $i^{\prime}$. Therefore, we have that case (a) occurs iff $\lambda_{j}^{(i)}=\lambda_{j-1}^{(i)}$ for some $i \geqslant j \geqslant 3$ and case (b) occurs iff $\lambda_{2}^{(i)}=\lambda_{1}^{(i)}$ for some $i \geqslant 2$.

\section{Proof of Identity 10}

\subsection{Connection with Gessel-Viennot}

We will call the elements in $\mathcal{V}_{\gamma}$ non-intersecting triangular tableaux compatible with the vector $\gamma$. The R.H.S. of the equation in Identity 10 will be denoted by $\Sigma(\gamma)$. Our goal is thus to show that $\Sigma(\gamma)=\prod_{1 \leqslant j<i \leqslant m}\left(a_{i}+1-a_{j}\right)$.

We will say that a partition $\lambda$ of length $i$ is compatible with $\gamma \in\{0,1\}^{m-1}$ if every part of $\lambda$ is not larger than $m$ and if $\lambda_{j+1}>\#\left\{k \leqslant j \mid \gamma_{k}=1\right\}$ for all $j=1, \ldots, \ell(\lambda)-1$. In this case, we will say that entry $j$ is critical in $\lambda$ if $\ell(\lambda) \geqslant j \geqslant 2$ and $\lambda_{j}=\lambda_{j-1}$. The weight of $\lambda$ will then simply be

$$
w(\lambda)=\prod_{j \text { critical }}\left(a_{\lambda_{j}}+b_{j-1}\right) .
$$

Note that the $a_{i}$ 's and $b_{j}$ 's are variables that are related such as described in Identity 10.

We denote by $P_{j, i}(\gamma)$ the sum of weights of partitions of length $i$, whose first part is equal to $j$, and that are compatible with $\gamma$. We define the $m$ by $m$ matrix $M(\gamma)$ as

$$
(M(\gamma))_{j, i}=P_{j, i}(\gamma)
$$

A triangular tableau $R$ compatible with the partition $\gamma$ is a sequence $\left(\lambda^{(1)}, \ldots, \lambda^{(m)}\right)$ of $m$ partitions compatible with $\gamma$ such that $\lambda^{(i)}$ is of length $i$ and such that $\sigma_{R}=\left[\lambda_{1}^{(1)}, \ldots, \lambda_{1}^{(m)}\right]$ is a permutation of $S_{m}$. The weight of a triangular tableau $R$ is

$$
w(R)=\operatorname{sign}\left(\sigma_{R}\right) \prod_{i=1}^{m} w\left(\lambda^{(i)}\right) .
$$

We denote by $\Sigma_{p i}(\gamma)$ the weighted sum of all the (possibly intersecting) triangular tableaux compatible with $\gamma$. 
Lemma 11. For any sequence $\gamma \in\{0,1\}^{m-1}$, we have

$$
\Sigma(\gamma)=\Sigma_{p i}(\gamma)=\operatorname{det} M(\gamma)
$$

For readers familiar with the Lindström-Gessel-Viennot lemma (LGV-lemma) [5], we remark that Lemma 11 is an instance of the LGV-lemma. Indeed, there is an interpretation of Lemma 11 in terms of "system of paths" in a directed acyclic graph depending on $\gamma$ where each row corresponds to one path. For the sake of simplicity we choose to reproduce the proof of the general LGV-lemma in terms of our objects instead of giving an explicit bijection preserving weights with system of paths of the ad hoc graph.

Proof. From the definition of a determinant, we have

$$
\operatorname{det} M(\gamma)=\sum_{\sigma \in S_{m}} \operatorname{sign}(\sigma) \prod_{i=1}^{m} P_{\sigma(i), i}(\gamma)
$$

Then, from the definition of $P_{j, i}(\gamma)$, we obtain

$$
\operatorname{det} M(\gamma)=\sum_{\sigma \in S_{m}} \operatorname{sign}(\sigma) \prod_{i=1}^{m}\left(\sum_{\lambda^{(i)} \text { of length } i \text { and } \lambda_{1}^{(i)}=\sigma(i)} w\left(\lambda^{(i)}\right)\right) .
$$

After expanding the product of the $m$ inner sums we recognize the weighted sum of triangular tableaux compatible with $\gamma$. Hence

$$
\operatorname{det} M(\gamma)=\Sigma_{p i}(\gamma)
$$

We describe a sign-reversing involution $\Phi$ on the set intersecting tableaux compatible with $\gamma$ to conclude that $\Sigma(\gamma)=\Sigma_{p i}(\gamma)$. Let $R=\left(\lambda^{(1)}, \ldots, \lambda^{(m)}\right)$ be such a tableau. Let $j_{R}$ be the index of the first column where at least one entry occurs at least twice. Let $i_{R}$ be the shortest row in which such an entry $x_{R}$ occurs in column $j_{R}$. Let $k_{R}$ be the next shortest row in which $x_{R}$ occurs in column $j_{R}$. We define $\Phi(R)=T=\left(\tau^{(1)}, \ldots, \tau^{(m)}\right)$ by $\tau_{j}^{\left(i_{R}\right)}=\lambda_{j}^{\left(k_{R}\right)}$ and $\tau_{j}^{\left(k_{R}\right)}=\lambda_{j}^{\left(i_{R}\right)}$ if $j<j_{R}$, otherwise $\tau_{j}^{(i)}=\lambda_{j}^{(i)}$. In other words $\Phi$ corresponds to the exchange of the entries in row $i_{R}$ and $k_{R}$ in all the columns whose index is strictly lower than $j_{R}$. Moreover $\Phi$ preserves $j_{R}, i_{R}, x_{R}$ and $k_{R}$ so $\Phi$ is an involution. It remains to check that $T$ is a triangular tableau compatible with $\gamma$ such that $w(T)=-w(R)$. By definition of a triangular tableau, the first column is a permutation thus $j_{R}>1$ so $\sigma_{T}$ is the appropriate composition of $\sigma_{R}$ by the transposition exchanging $i_{R}$ and $k_{R}$. This implies that $\operatorname{sign}\left(\sigma_{T}\right)=-\operatorname{sign}\left(\sigma_{R}\right)$. The rows of $T$ remain partitions because the two exchanged entries in column $j_{R}-1$ are not smaller than the common value $x_{R}$ in column $j_{R}$ of the corresponding rows. Finally, it is easy to see that the weight of $R$ and $T$ are the same. First observe that by construction the contribution to the weight coming from the critical entries smaller than $j_{R}$ is the same in $\tau^{\left(i_{R}\right)}$ (resp. $\tau^{\left(k_{R}\right)}$ ) and $\lambda^{\left(k_{R}\right)}$ (resp. $\lambda^{\left(i_{R}\right)}$ ). Similarly, the contribution to the weight coming from the critical 


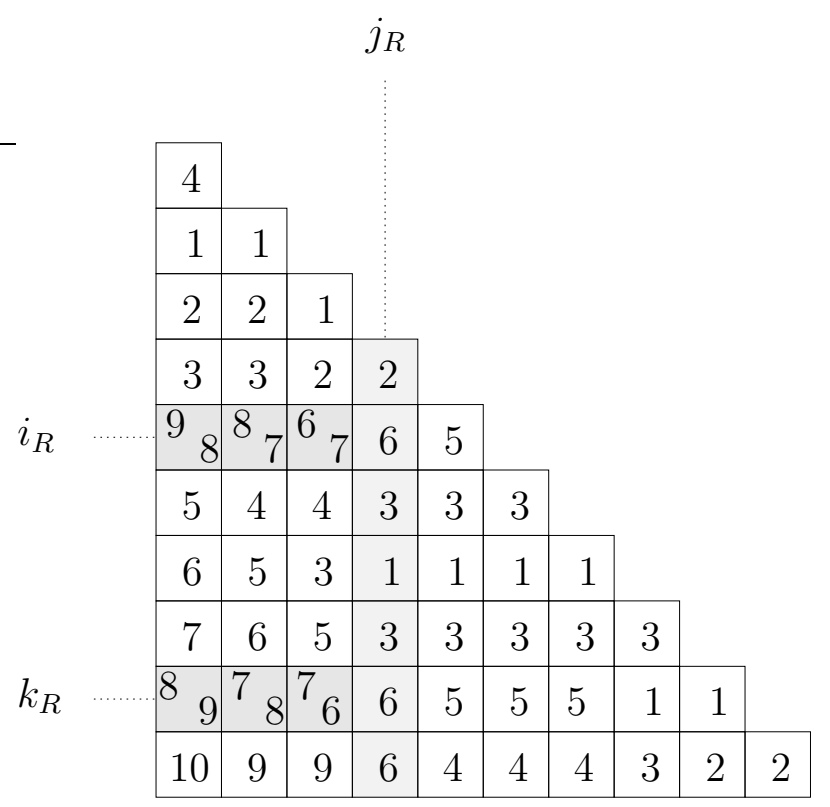

Figure 6: The bijection $\Phi$ illustrated with an example. The triangular tableaux $R$ and $T$ are represented on the same diagram. Labels of $R$ and $T$, when distinct, are in the upper left corner and lower right corner respectively. For the sake of simplicity we chose $\gamma=0^{m-1}$.

entries larger than $j_{R}$ is the same in $\tau^{\left(i_{R}\right)}$ (resp. $\tau^{\left(k_{R}\right)}$ ) and $\lambda^{\left(i_{R}\right)}$ (resp. $\lambda^{\left(k_{R}\right)}$ ). The result then follows since the possible critical entry $j_{R}$ in $\tau^{\left(i_{R}\right)}$ (resp. $\tau^{\left(k_{R}\right)}$ ) and in $\lambda^{\left(k_{R}\right)}$ (resp. $\lambda^{\left(i_{R}\right)}$ ) would give the same contribution to the weight given that the $j_{R^{-}}$th entry in both partitions is $x_{R}$.

We will first give a proof of the identity in the case $\gamma^{0}=(0, \ldots, 0) \in\{0,1\}^{m-1}$; we will do so by computing the determinant of $M\left(\gamma^{0}\right)$ by elementary row operations using certain technical results that we establish in the next subsection. From this particular case we will then be able to prove the result for an arbitrary $\gamma \in\{0,1\}^{m-1}$.

\subsection{Technical results}

Let $P_{j, i}^{[k]}$ be the sum of the weights of all partitions of length $i$ whose first part is $j$ and with at least one part equal to $j-l$ for each $l=1, \ldots, k$; we will use the notation $\mathcal{P}_{j, i}^{[k]}$ for this set of partitions. In particular, we have $P_{j, i}^{[0]}=P_{j, i}\left(\gamma^{0}\right)$ which are the entries of the matrix $M\left(\gamma^{0}\right)$.

We start with a lemma describing how to compute $P_{j, i}^{[k]}$ recursively; we introduce the notation $P_{j, i}^{[k],+}$ to stand for the result of the substitution $b_{1} \leftarrow b_{2}, b_{2} \leftarrow b_{3}, \ldots, b_{i-1} \leftarrow b_{i}$ in $P_{j, i}^{[k]}$. 
Lemma 12. Let $k \in \mathbb{N} . P_{j, i}^{[k]}=0$ if $j \leqslant k$ or $i \leqslant k$, and $P_{j, 1}^{[0]}=1$ for $j>0$. Otherwise,

$$
\begin{aligned}
& P_{j, i}^{[0]}=\left(a_{j}+b_{1}\right) \cdot P_{j, i-1}^{[0],+}+P_{j-1, i-1}^{[0],+}+\left[P_{j-1, i}^{[0]}-\left(a_{j-1}+b_{1}\right) P_{j-1, i-1}^{[0],+}\right], \\
& P_{j, i}^{[k]}=\left(a_{j}+b_{1}\right) \cdot P_{j, i-1}^{[k],+}+P_{j-1, i-1}^{[k-1],+} \quad \text { for } k \geqslant 1 .
\end{aligned}
$$

Proof. The first part of the lemma is obvious given that $\mathcal{P}_{j, i}^{[k]}$ is empty when $j \leqslant k$ or $i \leqslant k$, and that $\mathcal{P}_{j, 1}^{[0]}$ contains only the partition $[j]$ of weight 1 .

For the first recurrence formula, the three terms correspond to the subsets of $\mathcal{P}_{j, i}^{[0]}$ consisting of partitions whose second part has respectively size $j, j-1$, or some $l<j-1$. This latter term is equal to the weighted sum of the elements of $\mathcal{P}_{j-1, i}^{[0]}$ whose second part is different from $j-1$.

As for the second recurrence formula, the two terms correspond simply to the subsets of $\mathcal{P}_{j, i}^{[k]}$ made out of partitions whose second part has respectively size $j$ and $j-1$.

Let $\Delta_{j, i}^{[k]}$ be the difference $P_{j, i}^{[k]}-P_{j-1, i}^{[k]}$. The main result is then the following:

Proposition 13. For $k \in \mathbb{N}, i>k$ and $j>k+1$, we have

$$
\Delta_{j, i}^{[k]}=\left(a_{j}+1-a_{j-k-1}\right) P_{j, i}^{[k+1]}
$$

Proof. We will prove this relation by induction on $k$.

Case $k=0$; by reorganizing terms in the first recurrence formula of Lemma 12, we obtain

$$
\Delta_{j, i}^{[0]}=\left(a_{j}+b_{1}\right) \cdot \Delta_{j, i-1}^{[0],+}+\left(a_{j}+1-a_{j-1}\right) \cdot P_{j-1, i-1}^{[0],+},
$$

where $\Delta_{j, i}^{[k],+}$ is naturally defined in general as the result of the substitutions $b_{l} \leftarrow b_{l+1}$ in $\Delta_{j, i}^{[k]}$. We may assume by induction on $i$, that the case $k=0$ of the proposition is true for $\Delta_{j, i-1}^{[0],+}$ (the case $i=1$ being trivial); we thus get

$$
\Delta_{j, i}^{[0]}=\left(a_{j}+1-a_{j-1}\right) \cdot\left[\left(a_{j}+b_{1}\right) P_{j, i-1}^{[1],+}+P_{j-1, i-1}^{[0],+}\right] .
$$

Here the second factor on the right hand side is then equal to $P_{j, i}^{[1]}$ by Lemma 12 . This proves the proposition in the case $k=0$.

Case $k>0$; suppose the proposition is true for $k-1$. This gives

$$
\begin{aligned}
\Delta_{j, i}^{[k]} & =\left(a_{j}+b_{1}\right) \cdot \Delta_{j, i-1}^{[k],+}+\left(a_{j}-a_{j-1}\right) \cdot P_{j-1, i-1}^{[k],+}+\Delta_{j-1, i-1}^{[k-1],+} \\
& =\left(a_{j}+b_{1}\right)\left(a_{j}+1-a_{j-k-1}\right) P_{j, i-1}^{[k+1],+}+\left[\left(a_{j}-a_{j-1}\right)+\left(a_{j-1}+1-a_{j-k-1}\right)\right] P_{j-1, i-1}^{[k],+} \\
& =\left(a_{j}+1-a_{j-k-1}\right) \cdot\left[\left(a_{j}+b_{1}\right) P_{j, i-1}^{[k+1],+}+P_{j-1, i-1}^{[k],+}\right]
\end{aligned}
$$

The first equality comes from Lemma 12, the second by induction on $i$ for $\Delta_{j, i-1}^{[k],+}$ and by the induction hypothesis for $\Delta_{j-1, i-1}^{[k-1],+}$. We then recognize $P_{j, i}^{[k+1]}$ on the right hand side thanks to Lemma 12 again. The proof is then complete.

This recursive proof of Proposition 13 does not really explain the simplicity of its result; for this, we found a bijective proof, that is given in the Appendix. 


\subsection{Proof of the $\gamma^{0}$ case}

Let us consider the matrix $M\left(\gamma^{0}\right)=\left(P_{j, i}^{[0]}\right)$, whose determinant we have to compute since, from Lemma 11, we have $\Sigma\left(\gamma^{0}\right)=\operatorname{det}\left(M\left(\gamma^{0}\right)\right)$.

Let us first perform on $M\left(\gamma^{0}\right)$ the elementary row operations $L_{j} \leftarrow L_{j}-L_{j-1}$ with $j=m, m-1, \ldots, 2$, in this order. The coefficients that appear in rows 2 to $m$ then correspond to $\Delta_{j, i}^{[0]}$ for $j>1$. By Proposition 13, we have that for $j>1$ the quantity $a_{j}+1-a_{j-1}$ is a factor of every coefficient in row $j$.

So $\operatorname{det}\left(M\left(\gamma^{0}\right)\right)=\prod_{j>1}\left(a_{j}+1-a_{j-1}\right) \operatorname{det}\left(M^{[1]}\right)$, where the entries of $M^{[1]}$ are given by

$$
m_{j i}^{[1]}= \begin{cases}P_{j, i}^{[0]} & \text { for } j=1 \\ P_{j, i}^{[1]} & \text { for } j>1\end{cases}
$$

We repeat the operations $L_{j} \leftarrow L_{j}-L_{j-1}$ for $j=m, m-1, \ldots, 3$ on $M^{[1]}$. Coefficients $\Delta_{j, i}^{[1]}$ then appear in rows 3 and below. This implies that the quantities $a_{j}+1-a_{j-2}$ are factors of the determinant for $j=m, m-1, \ldots, 3$. Factorizing these quantities we obtain a new matrix $M^{[2]}$. One naturally applies this process successively, using Proposition 13 at each step, to obtain matrices $M^{[3]}, \ldots, M^{[m-1]}$. At the final stage we get by induction that

$$
\operatorname{det}\left(M\left(\gamma^{0}\right)\right)=\prod_{i>j}\left(a_{i}+1-a_{j}\right) \times \operatorname{det}\left(M^{[m-1]}\right)
$$

where the coefficient $(j, i)$ of $M^{[m-1]}$ is $m_{j i}^{[m-1]}=P_{j, i}^{[j-1]}$.

Now for $j>i, P_{j, i}^{[j-1]}$ is 0 by Lemma 12 ; and for $i=j, m_{i i}^{[m-1]}=P_{i, i}^{[i-1]}$, which is the weighted enumeration of $\mathcal{P}_{i, i}^{[i-1]}$. But this last set is easily seen to contain just one element, namely $(i, i-1, \ldots, 1)$ which has weight 1 . So $M^{[m-1]}$ is upper triangular with 1 's on the diagonal, and has consequently a determinant equal to 1 . This completes the proof of Identity 10 in the case of $\gamma^{0}$.

\subsection{Proof of the general case}

Let us fix $\gamma \in\{0,1\}^{m-1}$. We now have to prove that $\operatorname{det} M(\gamma)=\prod_{1 \leqslant j<i \leqslant m}\left(a_{i}+1-a_{j}\right)$. Define $\Gamma_{k}=\sum_{l=1}^{k-1} \gamma_{l}$, and let $\mathcal{P}_{j, i}(\gamma)$ be the set of partitions of length $i$ with first part $j$ that are compatible with $\gamma$. Let $\lambda$ be a partition in $\mathcal{P}_{j, i}\left(\gamma^{0}\right)$. Considering $\lambda$ as a word in the alphabet $\{1, \ldots, n\}$, we can define the factorization $\lambda=p_{\gamma}(\lambda) \cdot s_{\gamma}(\lambda)$, where $p_{\gamma}(\lambda)$ is the longest prefix that is compatible with $\gamma$, and $s_{\gamma}(\lambda)$ is the remaining suffix. We then fix an integer $k \in\{1, \ldots, m\}$, and consider the partitions $\lambda$ such that $p_{\gamma}(\lambda)$ has length $k$. In this case $p_{\gamma}(\lambda)$ belongs to $\mathcal{P}_{j, k}(\gamma)$, which implies that its last part is not smaller than $\Gamma_{k}+1$, while $s_{\gamma}(\lambda)$ is a partition of length $i-k$ whose first part is not larger than $\Gamma_{k+1}$; we will denote by $\mathcal{S}_{k, i}(\gamma)$ the latter set. It is clear that, conversely, the concatenation of an element of $\mathcal{P}_{j, k}(\gamma)$ with an element of $\mathcal{S}_{k, i}(\gamma)$ gives an element $\lambda$ of $\mathcal{P}_{j, i}\left(\gamma^{0}\right)$ with a prefix 
$p_{\gamma}(\lambda)$ of length $k$ (this gives indeed a decreasing sequence since $\gamma_{k} \in\{0,1\}$ implies that $\left.\Gamma_{k}+1 \geqslant \Gamma_{k+1}\right)$. In other words, the factorization above is a bijection between the partitions of $\mathcal{P}_{j, i}\left(\gamma^{0}\right)$ with a prefix $p_{\gamma}(\lambda)$ of length $k$, and the cartesian product $\mathcal{P}_{j, k}(\gamma) \times \mathcal{S}_{k, i}(\gamma)$.

We now consider the behavior of this bijection with respect to the weight $w$ attached to $\gamma$. The sum of the weights of $\mathcal{P}_{j, k}(\gamma)$ is by definition $P_{j, k}(\gamma)$. Let $S_{k, i}(\gamma)$ denote the weighted sum of $\mathcal{S}_{k, i}(\gamma)$ (here we need to index the partitions $\mu$ in $\mathcal{S}_{k, i}(\gamma)$ as $\left(\mu_{k+1}, \ldots, \mu_{i}\right)$, and the weight is then defined as before). We notice that $S_{k, i}(\gamma)=0$ if $k>i$ since $\mathcal{S}_{k, i}(\gamma)$ is empty in this case, and that $S_{i, i}(\gamma)=1$ since $\mathcal{S}_{i, i}(\gamma)$ consists only of the empty word. Finally, we have to study the weight attached to $\left(\lambda_{k}, \lambda_{k+1}\right)$, i.e. when one part belongs to $p_{\gamma}(\lambda)$ and the other to $s_{\gamma}(\lambda)$. If these two parts are different then the weight is 1 . Otherwise, by definition of the factorization, we are necessarily in the situation where $\lambda_{k}=\lambda_{k+1}=\Gamma_{k+1}$ and $\gamma_{k}=1$. The weight is then $a_{\Gamma_{k+1}}+b_{k}=1$ in this case also because of the relation $b_{k}=1-a_{\Gamma_{k+1}}$ (cf. the hypotheses in Identity 10).

In summary, we showed that the weighted sum $P_{j, i}\left(\gamma^{0}\right)$, under the specializations $b_{k}=1-a_{\Gamma_{k+1}}$ for all indices $k$ such that $\gamma_{k}=1$, is equal to $\sum_{k=1}^{m} P_{j, k}(\gamma) S_{k, i}(\gamma)$. If we define $M^{\prime}\left(\gamma^{0}\right)$ as the matrix $M\left(\gamma^{0}\right)$ under these specializations, and $S(\gamma)$ as the matrix $\left(S_{j, i}(\gamma)\right)_{j, i}$, this can be rephrased as

$$
M^{\prime}\left(\gamma^{0}\right)=M(\gamma) S(\gamma)
$$

from which we get

$$
\operatorname{det} M^{\prime}\left(\gamma^{0}\right)=\operatorname{det} M(\gamma) \cdot \operatorname{det} S(\gamma)=\operatorname{det} M(\gamma) \text {, }
$$

since $S(\gamma)$ is triangular with 1's on the diagonal. But we already computed $\operatorname{det} M\left(\gamma^{0}\right)$, which actually does not depend on the $b_{k}$ 's. This implies immediately that $\operatorname{det} M^{\prime}\left(\gamma^{0}\right)=$ $\operatorname{det} M\left(\gamma^{0}\right)$, since the former is defined as a specialization of the latter at certain values of the $b_{k}$ 's. We finally conclude that $\operatorname{det} M(\gamma)=\operatorname{det} M\left(\gamma^{0}\right)=\prod_{1 \leqslant j<i \leqslant m}\left(a_{i}+1-a_{j}\right)$ for any $\gamma \in\{0,1\}^{m-1}$, which completes the proof of Identity 10 .

Remark 14. Letting $\gamma^{1}=(1,1 \ldots, 1)$, it is easy to prove that $\Sigma\left(\gamma^{1}\right)=\prod_{1 \leqslant j<i \leqslant m}\left(a_{i}+\right.$ $1-a_{j}$ ), since there is only one vector $\left(\lambda^{(1)}, \ldots, \lambda^{(m)}\right)$ in $\mathcal{V}_{\gamma^{1}}$ (namely the one where $\lambda^{(i)}$ is the partition consisting of $i$ parts of size $i$ ). Thus, using the argument given in the proof of the general case, we get that $\operatorname{det} M\left(\gamma^{0}\right)$ at special values of the $b_{i}$ 's is equal to $\operatorname{det} M\left(\gamma^{1}\right)=\Sigma\left(\gamma^{1}\right)=\prod_{1 \leqslant j<i \leqslant m}\left(a_{i}+1-a_{j}\right)$. We would therefore immediately have that $\operatorname{det} M\left(\gamma^{0}\right)=\prod_{1 \leqslant j<i \leqslant m}\left(a_{i}+1-a_{j}\right)$ if we could simply prove that $\operatorname{det} M\left(\gamma^{0}\right)$ does not depend on the $b_{i}$ 's. Unfortunately, we did not find a way to prove this without computing the whole determinant.

Remark 15. A remark for those familiar with divided difference operators and Schubert calculus. After this paper was submitted, A. Lascoux showed us a proof of the $\gamma^{0}$ case based on these operators. In his approach, the result follows from the fact that $\operatorname{det} M\left(\gamma^{0}\right)$ can be shown to be an alternant under the action of the symmetric group provided by $\sigma_{j}+\partial_{j}$, where $\sigma_{j}$ and $\partial_{j}$ are respectively the interchange and divided difference operators on the variables $a_{j}$ and $a_{j+1}$ (see for instance [6]). The main step in this approach is to 
show that $\left(\sigma_{j}+\partial_{j}\right) P_{j, i}\left(\gamma^{0}\right)=P_{j+1, i}\left(\gamma^{0}\right)$ for all $i=1, \ldots, m$ and all $j=1, \ldots, m-1$, which can be done in a manner combinatorially very similar to computing the image of a certain double Schubert polynomial under $\partial_{j}$.

Acknowledgments. We are grateful to Sylvie Corteel for her interest in Identity 1, and especially for having presented the identity to PN. We thank Alain Lascoux for showing us his approach based on Schubert calculus mentionned in Remark 15.

\section{References}

[1] D. Bernard, M. Gaudin, F.D.M. Haldane and V. Pasquier, Yang-Baxter equations in long-range interacting systems, J. Phys. A: Math. Gen. 26, 5219-5236 (1993).

[2] P. Desrosiers, L. Lapointe and P. Mathieu, Jack polynomials in superspace, Comm. Math. Phys. 242, 331-360 (2003).

[3] P. Desrosiers, L. Lapointe and P. Mathieu, Orthogonality of Jack polynomials in superspace, Adv. Math. 212, 361-388 (2007).

[4] F. Knop and S. Sahi, A recursion and a combinatorial formula for the Jack polynomials, Invent. Math. 128, 9 (1997).

[5] I. Gessel and X. Viennot, Determinants, paths and plane partitions, preprint (1989). http://people.brandeis.edu/ gessel/homepage/papers/pp.pdf, or http://web.mac.com/xgviennot/iWeb/Xavier_Viennot/articles_files/determinant_89.pdf

[6] A. Lascoux, Yang-Baxter graphs, Jack and Macdonald polynomials, Ann. Comb. 5, 397-424 (2001).

[7] I. G. Macdonald, Symmetric Functions and Hall Polynomials, 2nd edition, Clarendon Press, Oxford, 1995.

[8] E. Opdam, Harmonic analysis for certain representations of graded Hecke algebras, Acta Math. 175, 75-121 (1995). 


\section{A A bijective proof of Proposition 13}

We will prove Proposition 13 bijectively in the following equivalent form:

Proposition 16. For $k \in \mathbb{N}, i>k$ and $j>k+1$, we have

$$
\left(a_{j}-a_{j-k-1}\right) P_{j, i}^{[k+1]}=\left(P_{j, i}^{[k]}-P_{j, i}^{[k+1]}\right)-P_{j-1, i}^{[k]} .
$$

Proof. The proof relies on the introduction of a new object: for $k>0$, an extended partition is defined as a partition $\lambda=\left(\lambda_{1}, \ldots, \lambda_{i}\right) \in \mathcal{P}_{j, i}^{[k]}$ with a right or left arrow, where the right or left arrow is located between two successive parts $\lambda_{u}$ and $\lambda_{u+1}$ such that $\lambda_{u}>\lambda_{u+1}=\lambda_{u}-1 \geqslant j-k$. We say in this case that $u$ is the position of the arrow of the extended partition. For instance, associated to the partition $\mu=(6,6,5,5,5,4,2,2,1) \in$ $\mathcal{P}_{6,9}^{[2]}$ are the four extensions

$$
\begin{aligned}
& (6,6 \rightarrow 5,5,5,4,2,2,1),(6,6 \leftarrow 5,5,5,4,2,2,1) \\
& (6,6,5,5,5 \rightarrow 4,2,2,1),(6,6,5,5,5 \leftarrow 4,2,2,1)
\end{aligned}
$$

whose arrows are respectively in positions $2,2,5$ and 5 . We will naturally call left (respectively right) extended partitions those with an arrow oriented to the left (resp. to the right), and define $\mathcal{E} \mathcal{P}_{j, i}^{[k]}$ as the set of all extensions of partitions in $\mathcal{P}_{j, i}^{[k]}$. The weight of a left (resp. right) extension of $\lambda$ whose arrow is in position $u$ is by definition the weight of $\lambda$, multiplied by $\left(a_{\lambda_{u}}+b_{u}\right)$ (resp. $-\left(a_{\lambda_{u+1}}+b_{u}\right)$ ). The weights of the four extended partitions above are then $w(\mu)$ multiplied respectively by $-\left(a_{5}+b_{2}\right),\left(a_{6}+b_{2}\right),-\left(a_{4}+b_{5}\right)$ and $\left(a_{5}+b_{5}\right)$.

We will now show that both sides of Equation (15) are in fact equal to the weighted sum of $\mathcal{E P}_{j, i}^{[k+1]}$, by a double counting of this last set.

We consider all the extensions of a given partition $\lambda \in \mathcal{P}_{j, i}^{[k+1]}$. There are clearly $k+1$ left extensions and $k+1$ right extensions of $\lambda$; if $\left(u_{r}\right)_{r=0 \ldots k}$ are the possible positions for the arrows in $\lambda$, then the weighted sum of these $2(k+1)$ extensions is equal to

$$
w(\lambda)\left(\sum_{r=0}^{k}\left(a_{j-r}+b_{u_{r}}\right)+\sum_{r=0}^{k}-\left(a_{j-r-1}+b_{u_{r}}\right)\right)=w(\lambda)\left(a_{j}-a_{j-k-1}\right) .
$$

So we obtain indeed the L.H.S. of (15) as the total weight of $\mathcal{E} \mathcal{P}_{j, i}^{[k+1]}$; the proof that it is also equal to the R.H.S. of (15) is more involved.

First, we use a sign reversing involution $\Psi$ on a certain subset of these extended partitions. We say that an extended partitions $\vec{\lambda} \in \mathcal{E P}_{j, i}^{[k+1]}$ associated to $\lambda$ (and whose arrow is in position $u$ ) is bad if one of the following conditions is satisfied:

1. $\vec{\lambda}$ is a left extension, and there exists a $v \geqslant u+1$ such that $\lambda_{v}=\lambda_{v+1} \geqslant j-k-1$. 
2. $\vec{\lambda}$ is a right extension, and there exists a $v \leqslant u$ such that $\lambda_{v-1}=\lambda_{v}$.

For example, among the four extensions of the partition $\mu$ above, the first three are bad, and the last one is good (i.e. not bad). Consider now the following function $\Psi$ on bad extended partitions: if $\vec{\lambda}$ is a left extension, choose $v$ minimal in the previous definition; then $\Psi(\vec{\lambda})$ is defined as

$$
\left(\lambda_{1}, \ldots, \lambda_{u}, \lambda_{u+1}+1, \lambda_{u+2}+1, \ldots, \lambda_{v}+1 \rightarrow \lambda_{v+1}, \ldots, \lambda_{i}\right)
$$

And if $\vec{\lambda}$ is a right extension, choose $v$ maximal in the definition; $\Psi(\vec{\lambda})$ is then defined as

$$
\left(\lambda_{1}, \ldots, \lambda_{v-1} \leftarrow \lambda_{v}-1, \lambda_{v+1}-1, \ldots, \lambda_{u}-1, \lambda_{u+1}, \ldots, \lambda_{i}\right)
$$

It is then easy to see that $\Psi$ is well defined, is an involution, and that the weights of $\vec{\lambda}$ and $\Psi(\vec{\lambda})$ are opposite. So the weighted sum of $\mathcal{E} \mathcal{P}_{j, i}^{[k+1]}$ is equal to the sum restricted to the good extended partitions, and we thus need to show that this latter sum is indeed equal to the R.H.S. of (15). Notice that $\vec{\lambda} \in \mathcal{E P}_{j, i}^{[k+1]}$ is good iff it is a left extension and there is exactly one part in $\lambda$ of each of the sizes $\lambda_{u+1}, \ldots, j-k-1$, or it is a right extension and there is exactly one part in $\lambda$ of each of the sizes $j, \ldots, \lambda_{u}$.

There is a bijection $\Theta_{L}$ between good left extended partitions, and partitions in $\mathcal{P}_{j, i}^{[k]}$ with at least two equal parts of size superior to $j-k-1$, and no part of size $j-k-1$. $\Theta_{L}(\vec{\lambda})$ is obtained from $\vec{\lambda}$ by deleting the arrow, and increasing by one the parts $\lambda_{u+1}, \ldots, \lambda_{v}$, where $u$ is the position of the arrow and $v$ is such that $\lambda_{v}=j-k-1$. $\Theta_{L}$ is weight preserving, and the weight of its image can be written as

$$
\left(P_{j, i}^{[k]}-P_{j, i}^{[k+1]}\right)-L_{j, i}^{[k]},
$$

where $\left(P_{j, i}^{[k]}-P_{j, i}^{[k+1]}\right)$ is the weight of partitions in $\mathcal{P}_{j, i}^{[k]}$ with no part of size $j-k-1$, and $L_{j, i}^{[k]}$ gives the weights of partitions in $\mathcal{P}_{j, i}^{[k]}$ that have exactly one part of each of the sizes $j, \ldots, j-k$, and no part of size $j-k-1$.

Then, there is also a bijection $\Theta_{R}$ between good right extended partitions, and partitions in $\mathcal{P}_{j-1, i}^{[k]}$ with at least two equal parts of size between $j-k-1$ and $j-1$. $\Theta_{R}(\vec{\lambda})$ is obtained from $\vec{\lambda}$ by deleting the arrow and by loweringing by one the parts $\lambda_{1}, \ldots, \lambda_{u}$, where $u$ is the position of the arrow. $\Theta_{R}$ is weight reversing, and the weight of its image is

$$
P_{j-1, i}^{[k]}-R_{j-1, i}^{[k]},
$$

where $R_{j-1, i}^{[k]}$ is the weighted sum of the partitions in $\mathcal{P}_{j-1, i}^{[k]}$ that have exactly one part of each of the sizes $j-1, \ldots, j-k-1$.

Putting everything together, we have that the weighted sum of $\mathcal{E} \mathcal{P}_{j, i}^{[k+1]}$ is equal to its restriction to good partitions, which in turn is equal to (16) minus (17) thanks to the weight preserving bijection $\Theta_{L}$ and the weight reversing bijection $\Theta_{R}$. But we also have that $R_{j-1, i}^{[k]}=L_{j, i}^{[k]}$ through the weight preserving bijection that increases by 1 the first $k$ parts of a partition. Thus, we obtain indeed the R.H.S. of Equation (15) as the weighted sum of $\mathcal{E P}_{j, i}^{[k+1]}$, and the proof is complete. 